\title{
Dynamic aspects of design and maintenance of the rotating machinery applied in the mining industry
}

\author{
Tomasz Szolc ${ }^{1, *}$, Robert Konowrocki ${ }^{1}$, Andrzej Pochanke $^{2}$ and Maciej Michajłow ${ }^{1}$ \\ ${ }^{1}$ Institute of Fundamental Technological Research of the Polish Academy of Sciences, ul. Pawińskiego 5B, 02-106 Warsaw, Poland \\ ${ }^{2}$ Department of Electrical Engineering of the Warsaw University of Technology, Plac Politechniki 1, 00-661 Warsaw, Poland
}

\begin{abstract}
In the paper a dynamic behaviour of the selected typical group of rotating machines applied in the mining industry is investigated. These are the beater mills and crushers as well as blowers and compressors, all driven by the asynchronous motors. In particular, there is considered an influence of various types of machine working tool loadings on the system lateral steady-state dynamic responses as well as a mutual torsional electromechanical interaction between the driving motor and the driven machine in transient operational conditions. The theoretical calculations have been performed by means of the advanced structural mechanical models. The conclusions drawn from computational results can be very useful during design phase of these devices as well as helpful for their users during regular maintenance.
\end{abstract}

\section{Introduction}

An operation of many machines and devices applied in the mining industry is characterized by rotational motion of their fundamental working tools. To this broad group belong several types of beater mills and beater crushers used in main production processes as well as blowers, rotor pumps, compressors and others regarded as auxiliary units. Majority of them are driven by electric motors by means of flexible couplings and by spur- or planetary gears. The still increasing work efficiency requirements to these machines, commonly observed nowadays, result in more and more dynamic character of their nominal operation. Namely, all these machines transmit now greater and greater power, they work with bigger and bigger rotational speeds, with greater and greater flow velocities and pressures as well as with raising impact force values caused by the comminution processes. These operational performances cause high steady-state or transient dynamic over-loadings which usually excite several types of mechanical vibrations. These vibrations often lead to more or less detrimental exploitation consequences in the form of dangerous material fatigue defects and too fast tribological wear of the responsible structural elements as well as to harmful noise generation, transmission of damaging oscillations to the surrounding environment and to many others. Thus, these dynamic aspects and particularly the vibratory effects should be more and more seriously taken into consideration during design and maintenance phases of such machines, in addition to the common traditional engineering routines applied till present. This postulated modernized approach ought to reduce to thorough and complete dynamic analyses associated with experimental verifications in the frameworks of design and prototyping phases as well as to advanced on-line monitoring and fault detections during a regular maintenance. It is worth emphasizing that for this reason there should be applied modern mechatronic methods based on more and more advanced control and diagnostic algorithms using several sensors, controllers, actuators, transducers and other electronic devices.

As it follows from practical engineering observations and scientific literature studies, e.g. in [1-3], such modern approaches to design and maintenance are mainly practiced in the case of typical flow rotating machines, i.e. for compressors, turbines, pumps, fans and blowers. But very little attention for this purpose seems to be focused to beater mills and crushers which can be also regarded as classical rotor-machines, because of their highly dynamic character of operation. According to the above, in this paper some important aspects of dynamic analyses necessary to achieve a correct design and possibly trouble-free maintenance of the high-speed beater mills, crushers and other rotor-machines are going to be considered.

\section{Objects of investigations}

The dynamic investigations will be focused on the typical high-speed beater mills, beater and hammer crushers as well as to the medium-speed blowers commonly applied as auxiliary devices for the ball-, rodand roller-bowl mills. All these units are usually characterized by mutually similar structures. Namely, their working-tool parts consist of heavy beater wheels or impellers attached to the drive shaft in the form of an

\footnotetext{
* Corresponding author: tszolc@ippt.pan.pl
} 
overhung rotor creating in this way a rotor-shaft. This rotor-shaft is commonly supported by rolling bearings and driven by an electric motor by means of a flexible coupling only, but without any gear stage. The schematic view of such object is presented in Fig. 1. It is worth noting the characteristic feature of these devices, namely

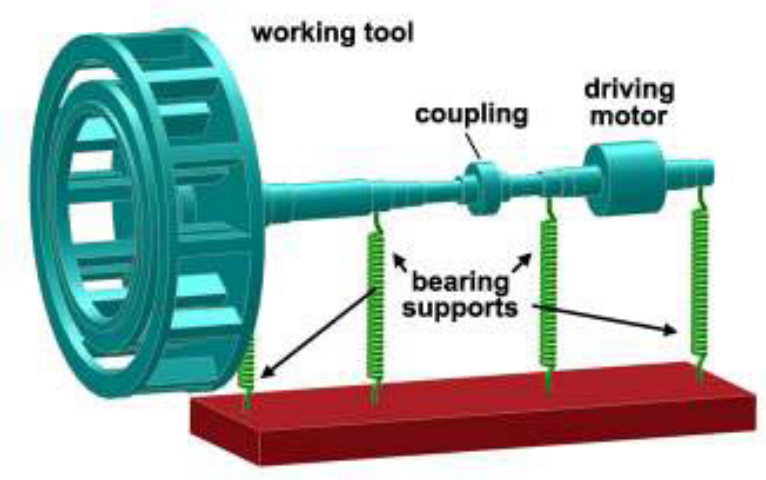

Fig. 1. Scheme of the rotor-machine.

a huge mass of the beater mill or impeller in comparison with the total mass of the entire unit as well as a much greater polar mass moment of inertia of the whole driven part than the polar mass moment of inertia of the driving electric motor rotor. These machines work with maximal rotational speeds reaching $3000 \mathrm{rpm}$ or even more. Due to the mentioned above features a character of their operation is highly dynamic, which results in excitation of bending, torsional and axial steady-state or transient vibrations, where the two former ones seem to be predominant. As it follows from fundamentals of applied mechanics and vibration theory, because of the abovementioned lack of gear stages in the considered devices, their bending and torsional vibrations can be investigated separately, since eventual possible weak couplings between them are negligible.

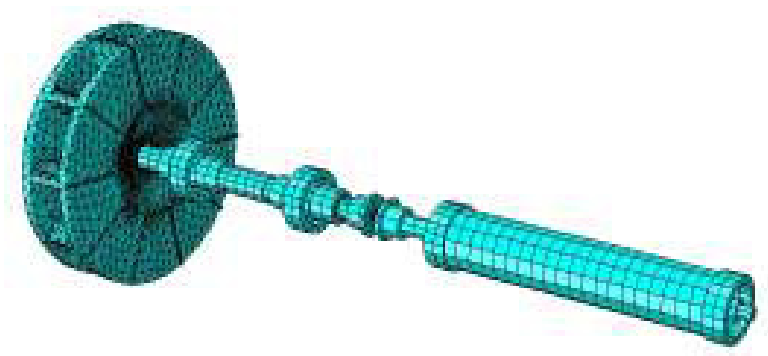

Fig. 2. The 3D FEM model of the rotor-machine.

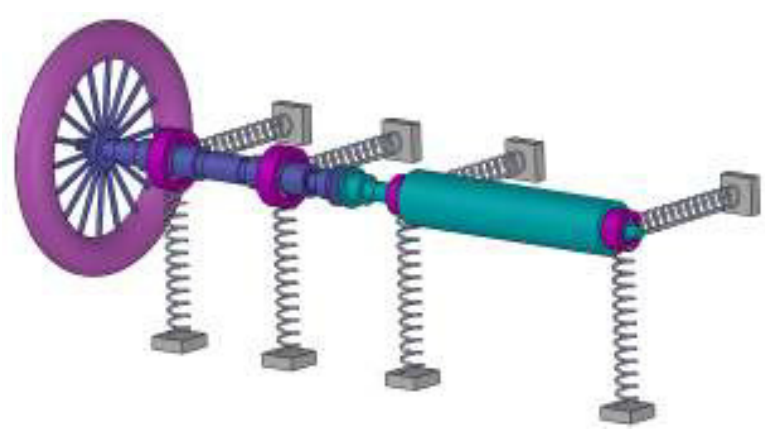

Fig. 3. The hybrid (beam) model of the rotor-machine.
For the aim of possibly reliable vibration analyses two structural mechanical models of the discussed objects have been assumed. These are the 3-D finite element model presented in Fig. 2 as well as the beam continuous finite element (hybrid) model shown in Fig. 3. Both of them thoroughly take into consideration system geometrical shapes, material parameters and bearing support visco-elastic properties. An expected mutual similarity of results of eigenvibration analyses and simulations of forced vibrations obtained by means of these models is a criterion of their computational reliability for practical engineering purposes.

\section{Investigation of bending vibrations}

The kinematic and elastic properties of the flexible coupling which connects the machine with the driving motor enable us to regard the driven shaft and the electric motor rotor as dynamically separated from the viewpoint of their lateral motions. Thus, for the aim of bending vibration analysis investigations can be focused on the driven machine part only, while the lateral dynamics problem of the electric motor can be assumed as more or less completely solved by a producer.

Here, the lateral motion, associated with bending vibrations of the rotor-shaft-bearing system represented by the two abovementioned structural models shown in Fig. 2 and 3, can be generally described by the following set of linear ordinary differential equations:

$$
\mathbf{M} \cdot \ddot{\mathbf{r}}(t)+\mathbf{C}(\Omega) \cdot \dot{\mathbf{r}}(t)+\mathbf{K}(\Omega) \cdot \mathbf{r}(t)=\mathbf{F}\left(t, \Omega^{2}\right),
$$

where:

$$
\mathbf{C}(\Omega)=\mathbf{C}_{0}+\Omega \cdot \mathbf{C}_{g}(\Omega) \text { and } \mathbf{K}(\Omega)=\mathbf{K}_{0}+\Omega \cdot \mathbf{K}_{d}(\Omega) .
$$

The symbols $\mathbf{M}$ and $\mathbf{K}_{0}$ denote respectively the mass and stiffness matrices, $\mathbf{C}_{0}$ is the damping matrix containing the damping coefficients of the bearing supports and $\mathbf{C}_{\mathrm{g}}(\Omega)$ denotes the skew-symmetrical matrix of gyroscopic effects. Anti-symmetrical effects due to the standard body material damping model of the rotating shaft are expressed by the skew-symmetrical matrix $\mathbf{K}_{d}(\Omega)$. The symbol $\mathbf{F}\left(t, \Omega^{2}\right)$ denotes the external excitation vector due to the dynamic loadings induced by the working process, unbalances and gravitational forces. In the case of $3 \mathrm{D}$ FEM model vector $\mathbf{r}(t)$ contains generalized coordinates. However, motion of the hybrid model is described by the modal coordinates contained in vector $\mathbf{r}(t)$ as well. The number of equations (1) corresponds to the total number of degrees of freedom of the FEM model or to the number of bending eigenmodes taken into consideration in the frequency range of interest, if the hybrid model is used.

It is worth reminding that bending natural frequencies, eigenmodes and magnitudes of forced lateral vibrations of rotor-shaft systems are strongly dependent on visco-elastic properties of the bearing supports. Since the considered rotating object is assumed to be suspended on relatively hard rolling element bearings, a flexibility of the bearing housings and of 
their foundation must be thoroughly taken into consideration. For this purpose the finite element method has been applied for a three-dimensional modelling of these structures in order to determine their equivalent vertical and horizontal static and dynamic stiffness characteristics. The 3D-FEM model with 88561 degrees of freedom of the steel foundation frame of the entire unit is presented in Fig. 4. Here, the proportional 4\% damping of the hysteresis type has been assumed for further computations.

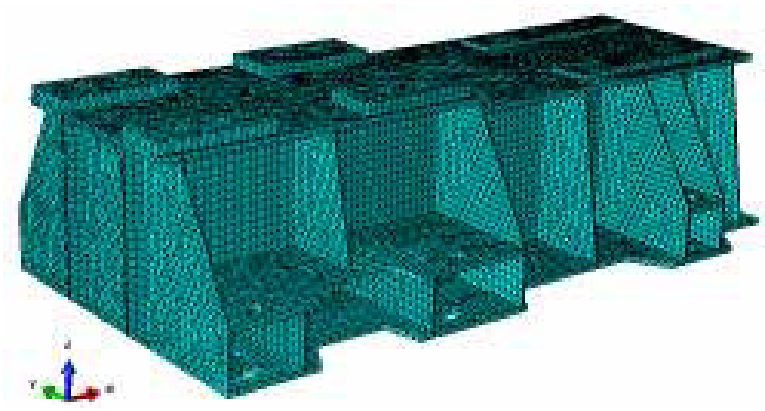

Fig. 4. The 3D FEM model of the machine foundation frame.

Since the 3D FEM model shown in Fig. 2 very precisely describes geometrical shapes of the beater wheel or blower impeller, but on the other hand is characterized by a relatively great number of degrees of freedom, i.e. exceeding 33000 , it has been applied as a reliable tool for eigenvibration analyses. For this purpose matrices $\mathbf{C}_{0}, \mathbf{K}_{d}(\Omega)$ and vector $\mathbf{F}\left(t, \Omega^{2}\right)$ could be omitted in Eqs. (1) for computations. Here, natural frequency values and mode shapes determined by means of this model were used as a benchmark for calculation of selected structural parameters of the hybrid model which is simpler, but much more effective for simulations of forced vibrations.

In order to investigate the qualitative dynamic properties of the considered rotating object, the eigenvalue problem had to be solved first by means of the two mechanical models described above. In Fig. 5 there are plotted the fundamental natural frequency characteristics expressed as rotational speed functions in the form of Campbell diagram with marked critical speeds corresponding to forward whirls of the successive eigenmodes. For a better clarity, on the left-hand side of this diagram also the respective bending eigenfunctions are depicted. As it follows from this figure, within the entire rotational speed range of the considered machine, i.e. $0-3000 \mathrm{rpm}$ corresponding to $0-50 \mathrm{rev} / \mathrm{s}$, three eigenmodes occur. Due to the overhung structure of the rotor-shaft, where the beater-wheel/impeller mass is equal to ca. $80 \%$ of the entire rotating mass, its first two eigenmodes are significantly sensitive to the gyroscopic effects with the natural frequency values within ca. 20$65 \mathrm{~Hz}$ for the first mode and within $100-145 \mathrm{~Hz}$ for the second one. However, the backward and forward precession branches of the third eigenform with the natural frequencies 415 and $419 \mathrm{~Hz}$ are almost independent of the shaft rotational speed, as shown in Fig. 5. It is worth noting here that the mutual differences

III
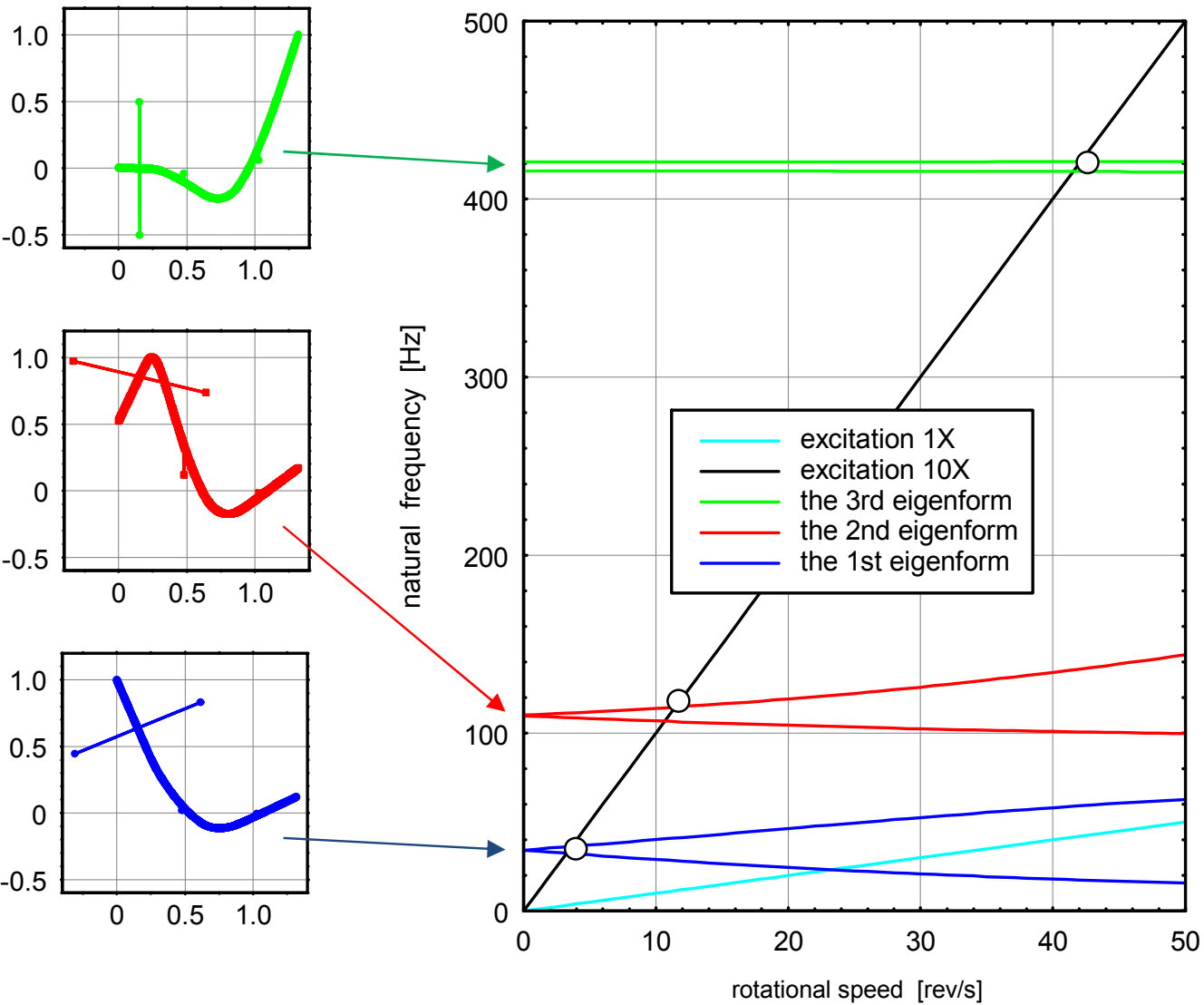

Fig. 5. The Campbell diagram for bending vibrations of the overhung rotor-shaft 


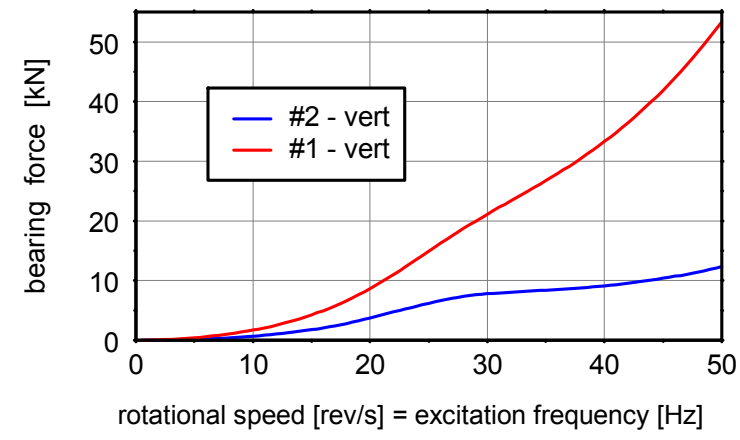

Fig. 6. Bearing force amplitude characteristics due to beater wheel unbalances.

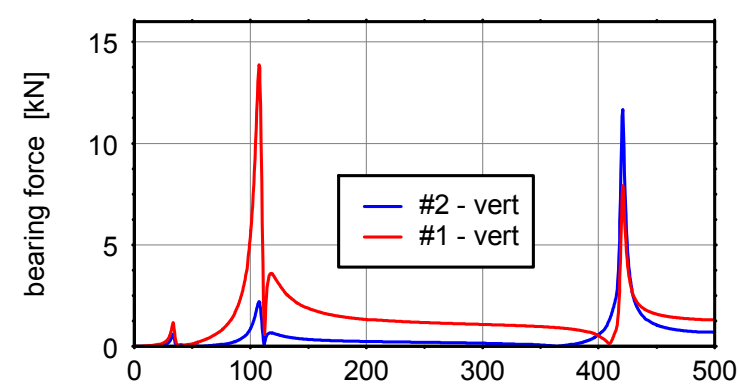

$10 \times$ rotational speed $[\mathrm{rev} / \mathrm{s}]]$ = excitation frequency $[\mathrm{Hz}]$

Fig. 7. Bearing force amplitude characteristics due to beater wheel operational dynamic loadings.

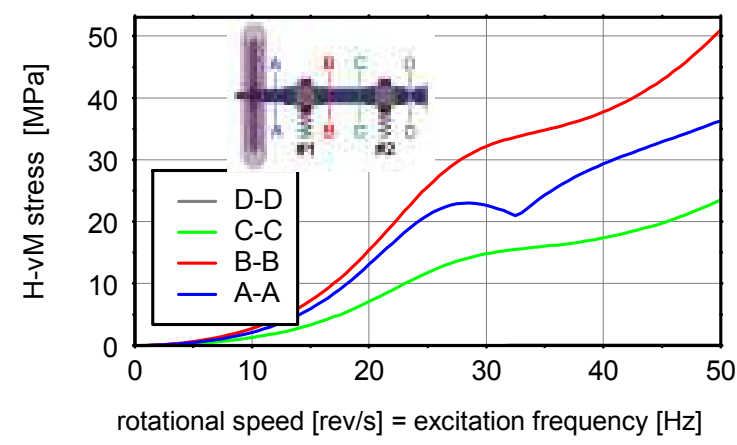

Fig. 8. Huber-von Mises stress amplitude characteristics due to beater wheel unbalances.

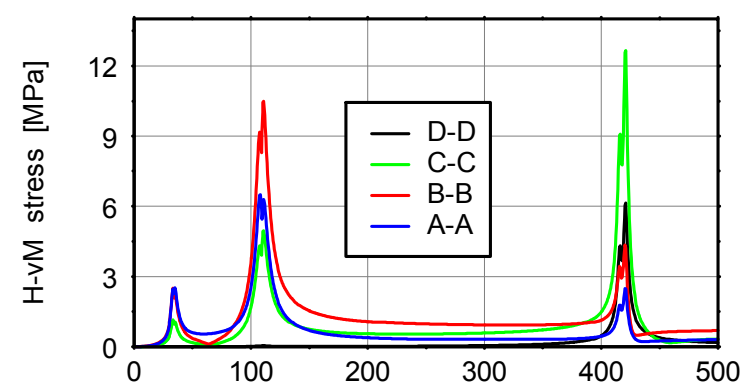

$10 \times$ rotational speed $[\mathrm{rev} / \mathrm{s}]]$ = excitation frequency $[\mathrm{Hz}]$

Fig. 9. Huber-von Mises stress amplitude characteristics due to beater wheel operational dynamic loadings.

\footnotetext{
*Corresponding author: tszolc@ippt.pan.pl
}

between the corresponding natural frequency values determined using the 3D-FEM model and by means of the hybrid one did not exceed $2.9 \%$. Moreover, form the obtained Campbell diagram it follows that the investigated machine operates as an under-critical rotor shaft within its entire rotational speed range under synchronous excitation of frequency $1 \mathrm{X}$. However, if an excitation of frequency $10 \mathrm{X}$, i.e. ten times rotational speed value, is assumed, e.g. due to an operation of the beater wheel with 10 beaters, then three critical speeds are observed, which can be a source of possible resonances, see Fig. 5.

According to the above, two types of external excitation for the investigated rotating machine will be assumed for the forced bending vibration analysis. These are: the synchronous harmonic excitation $1 \mathrm{X}$ in the frequency range $0-50 \mathrm{~Hz}$ and the harmonic excitation $10 \mathrm{X}$ with the frequency ten times greater than that following from the rotational speed in the entire range 0 $500 \mathrm{~Hz}$. The former can be caused not only by the beater mill residual unbalances, but also by a non-proportionate mass change of the beaters due to their fast wear or random adhesion of the comminuted raw material to the working surfaces. However, the latter type of excitation can be associated with the comminution process resulting in repeatable mutual impacts of the raw material particles to the beaters within each one full beater wheel rotation.

For the aim of possibly reliable assessment of a dynamic load affecting the investigated rotor-shaft system an equivalent unbalance of the beaterwheel/impeller is applied, which overestimates commonly admissible values in order to take into consideration additional effects caused by the abovementioned fast wear or random raw-material adhesion. Here, the magnitude of this equivalent unbalance has been assumed $\sim 18.5$ times greater than that following form an application of the ISO 1940-1, [4], and ISO 11342, [5], norms. In Figs. 6-9 there are plotted the amplitude-frequency characteristics in the form of Bode diagrams obtained for steady-state dynamic lateral responses of the rotor-shaft-bearing system. Fig. 6 and 7 present plots of bearing reaction force amplitudes, where in Fig. 6 the response due to the synchronous excitation $1 \mathrm{X}$ by the assumed equivalent unbalance is illustrated and in Fig. 7 there is shown the analogous response induced by the harmonic excitation of frequency $10 \mathrm{X}$ caused e.g. by the comminution dynamic effects. Respectively, for the same excitations $1 \mathrm{X}$ and $10 \mathrm{X}$ in Figs. 8 and 9 the Bode diagrams of the Huber - von Mises stress in the most responsible rotorshaft cross-sections are presented. The plots demonstrated in Fig. 6 and 8 indicate clearly undercritical character of the dynamic responses due to the synchronous excitation $1 \mathrm{X}$ within the admissible rotational speed range. This fact is qualitatively confirmed by the Campbell diagram shown in Fig. 5. Here, the bearing force- and stress amplitudes significantly raise with the rotational speed, but they do not reach a resonance peak with the first system eigenform. However, for the harmonic excitation 10X in Fig. 7 and 9 there are observed three resonance peaks 
corresponding to the successive three system eigenforms presented in Fig. 5. Nevertheless, it is worth to emphasize that the all extreme force and stress values of these peaks are respectively many times smaller than the maximal force and stress amplitudes presented in Figs. 6, 8 and obtained for the under-critical external excitation. This fact can be substantiated by a much greater sensitivity of the considered rotor-shaft system to relatively low-frequency harmonic excitations with amplitudes proportional to squares of the actual rotational speed values in comparison with the excitations characterized by correspondingly the same amplitudes and appropriately ten times higher excitation frequencies. In addition, it is to remember that structural damping of such mechanical systems attenuates more intensively extreme dynamic response magnitudes with greater fluctuation frequencies.

\section{Investigation of torsional vibrations}

Contrary to the bending vibration analysis performed above, for investigations of torsional oscillation problem for the studied object the entire system, i.e. including the electric motor rotor, must be considered. Namely here, the qualitative and quantitative elastic properties of the flexible coupling play very important role for an electromechanical interaction between the driving motor and the driven machine.

Here, torsional motion of the rotor-shaft-motor system represented by the two abovementioned structural models shown in Fig. 2 and 3 can be generally described by the following set of non-linear ordinary differential equations:

$$
\mathbf{M} \ddot{\mathbf{r}}(t)+\mathbf{C}(\Delta \theta(t)) \dot{\mathbf{r}}(t)+\mathbf{K}(\Delta \theta(t)) \cdot \mathbf{r}(t)=\mathbf{T}(t, \Omega(t), \theta(t)),
$$

where:

$$
\mathbf{C}(\Delta \theta(t))=\mathbf{C}_{0}+\mathbf{C}_{c}(\Delta \theta(t)), \mathbf{K}(\Delta \theta(t))=\mathbf{K}_{0}+\mathbf{K}_{c}(\Delta \theta(t)) .
$$

The symbols $\mathbf{M}, \mathbf{K}_{0}$ denote respectively the mass and stiffness matrices, $\mathbf{C}_{0}$ is the damping matrix and $\mathbf{C}_{\mathrm{c}}(\Delta \theta(t))$ denotes the matrix containing the non-linear damping coefficients of the flexible coupling, where $\Delta \theta(t)$ is the current coupling twist angle. The non-linear stiffness coefficients of this flexible coupling are contained matrix $\mathbf{K}_{c}(\Delta \theta(t))$. The symbol $\mathbf{T}(t, \Omega(\mathrm{t}), \theta(t))$ denotes the vector of external excitation due to the electromagnetic torque generated by the driving motor as well as due to retarding torques imposed to the driven machine working tool and expressed both as functions of the current rotational speed value $\Omega(\mathrm{t})$ and the system dynamic response $\theta(t)$. Similarly as before, in the case of 3D FEM model vector $\mathbf{r}(t)$ contains generalized coordinates and motion of the hybrid model is described by the modal coordinates contained in vector $\mathbf{r}(t)$ as well. Similarly as before, the number of equations (2) also corresponds to the total number of degrees of freedom of the FEM model or to the number of torsional eigenmodes taken into consideration in the frequency range of interest, when the hybrid model is used.
As already mentioned above, actual torsional stiffness values of the flexible coupling very essentially influence natural frequencies of the considered system. The flexible coupling applied here is non-linear with the following progressive characteristic determined by its producer and depicted in Fig. 10. In the investigated case, the fundamental first torsional natural frequency value significantly depends on the working point

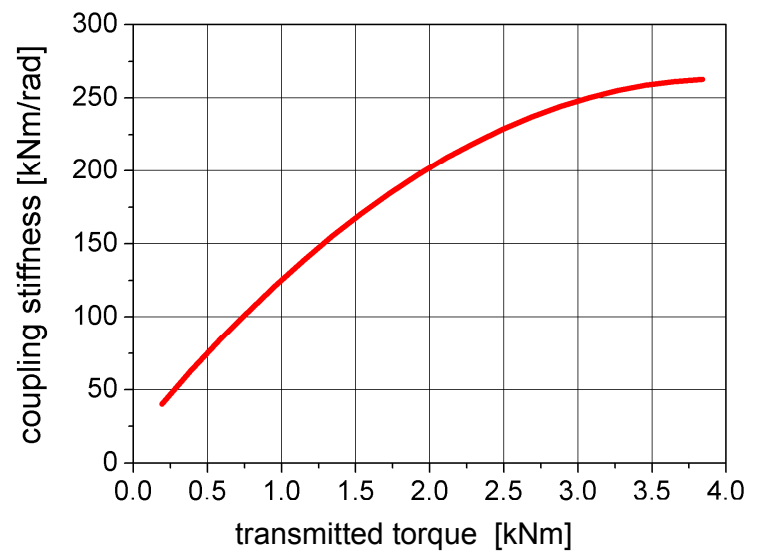

Fig. 10. Torsional stiffness characteristic of the flexible coupling.

determined by the current torque transmitted by this coupling, according to the characteristic presented in Fig. 10. Namely, for zero initial twist of the coupling the first natural frequency calculated by means of the 3DFEM model and using the hybrid model is equal to ca. $15 \mathrm{~Hz}$, where the mutual difference did not exceed $0.5 \%$ But for the maximal admissible torsional loading the appropriately increased coupling stiffness results in the first natural frequency exceeding $50 \mathrm{~Hz}$. As it will turn out later, this property shall have a significant influence on the system transient torsional vibrations. Nevertheless, within the entire range of twisting torques transmitted by this coupling, i.e. for all the respective first natural frequency values, the corresponding eigenmode function has the following shape presented in Fig. 11. This eigenfunction is characterized by the whole driven machine part (on the left hand side) almost equal to zero and by the motor part (on the right hand side) equal to $\sim-1$. This means that dynamic excitations imposed only to the motor rotor are able to induce torsional vibrations of the considered system. From the results of eigenproblem analysis performed by these two mechanical models it follows that the next eigenmodes occur above $500 \mathrm{~Hz}$ and they are very hardly excitable by dynamic retarding torques loading the beater-wheel or impeller. These features follow from the relatively large torsional stiffness of the overhung rotor-shaft structure as well as because in such machines the polar mass moment of inertia of the driven part is much greater than that of the driving motor rotor. In the studied case this ratio exceeds the value of 41 .

For the need of simulation of the rotor-machine torsional vibrations as well as in order to investigate electromechanical coupling effects in the considered system the properly advanced circuit model of the 


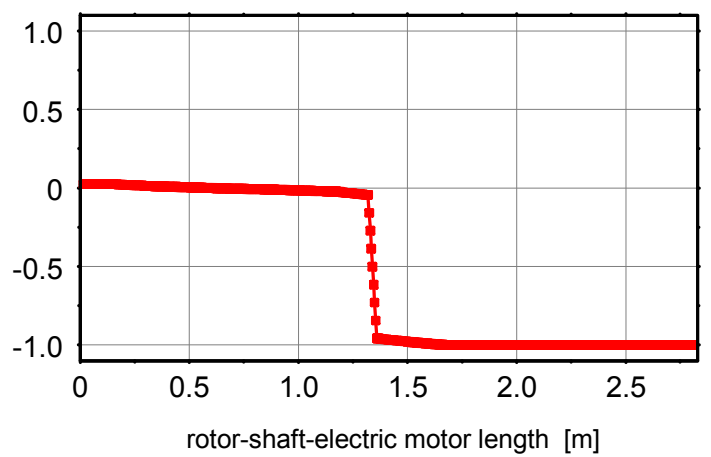

Fig. 11. The first torsional eigenmode function of the rotorshaft system.

electric motor seems to be sufficiently accurate. In the case of the symmetrical three-phase asynchronous motor electric current oscillations in its windings are described by the six circuit voltage equations. Then, according e.g. to [6], by the use of Clark's transformation they are reduced into four voltage equations in the so called ' $\alpha \beta$ ' reference system. This approach enables us more effective numerical computations without any loss of accuracy, together with a possibility of taking into consideration a variable resistance of the rotor windings as well as a convenient control of the motor operation. Then, the electromagnetic torque generated by such a motor can be expressed by the following formula:

$$
\begin{aligned}
T_{e l}=\frac{3}{2} p M & {\left[\cos (p \vartheta(t)) \cdot\left(i_{\beta}^{s} \cdot i_{\alpha}^{r}-i_{\alpha}^{s} \cdot i_{\beta}^{r}\right)-\right.} \\
& \left.-\sin (p \vartheta(t)) \cdot\left(i_{\alpha}^{s} \cdot i_{\alpha}^{r}-i_{\beta}^{s} \cdot i_{\beta}^{r}\right)\right],
\end{aligned}
$$

where $M$ denotes the relative rotor-to-stator coil inductance, $p$ is the number of pairs of the motor magnetic poles, $\vartheta(t)$ is the current rotor angular displacement including the average and vibratory component and $i_{\alpha}^{s}, i_{\beta}^{s}$ are the electric currents in the stator windings reduced to the electric field equivalent axes $\alpha$ and $\beta$ and $i_{\alpha}^{r}, i_{\beta}{ }^{r}$ are the electric currents in the rotor windings reduced to the electric field equivalent axes $\alpha$ and $\beta$, [6,7]. The static characteristics of the considered asynchronous motor, determined accoriding to $[7,8]$, are shown in Fig. 12. Here, the blue line

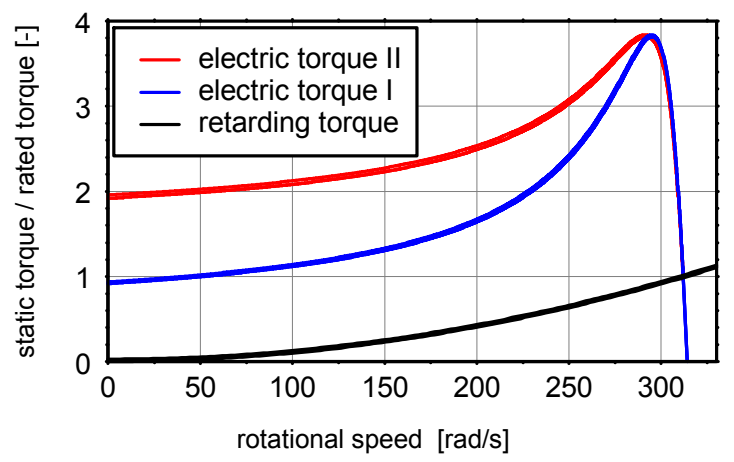

Fig. 12. Static characteristic of the asynchronous motor and of the retarding torque. corresponds to Variant I characterized by the smaller staring torque. By means of the red line there is plotted the characteristic for Variant II with the greater staring torque caused by an increased rotor resistance.

As it followed from results of introductory simulations, because of the abovementioned relatively huge mass moment of inertia of the machine working tool in the form of a beater wheel or blower/compressor impeller, and because of the correspondingly small magnitude of the retarding torque variable components, in steady-state operating conditions possible torsional dynamic excitations turn out to be negligible. Then, the entire object works under almost static twist. According to the above, the retarding torques generated by the working tool can be regarded as of the aerodynamic type and modelled by means of the parabolic functions of the current rotational speed values. However, in transient operating conditions, e.g. during start-ups, the considered rotating machine drive system can be affected by severe torsional vibrations excited by the electromagnetic torque generated by the asynchronous motor described by Eq. (3). It is particularly the case, when in order to decrease the machine price any inverters are applied. Then, each start-up has to be carried out using an usually troublesome dischargeable clutch. Otherwise, such run-ups may lead to too high overloadings and damage of the flexible coupling which connects the driven machine with the motor.

In order to illustrate such a situation, the introductory numerical simulations have been performed for classical start-ups of the considered machine, i.e. without any electronic control of the asynchronous motor. In the first computational example the stiffness characteristic of the flexible coupling has been linearized around the working point corresponding to the nominal steady-state operating conditions with the average rotational speed $2980 \mathrm{rpm}$. Then, the first torsional natural frequency of the machine drive system associated with the eigenmode function shown in Fig. 11 is equal to $34.3 \mathrm{~Hz}$. In Fig. 13 there are plotted time-histories of the system dynamic response due to the start-up from a standstill to the nominal operation. In this figure by the black line the time-history of the current rotational speed is marked. The red line corresponds to the electromagnetic motor torque and using the blue line there is illustrated a parabolic rise of the working tool retarding torque. The

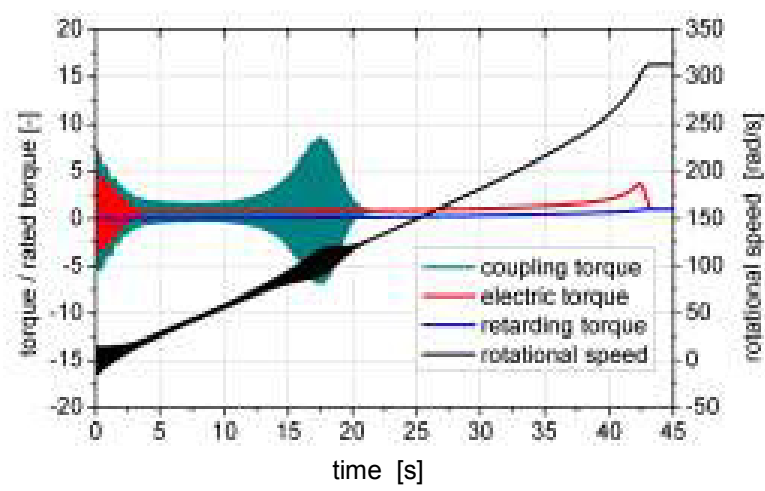

Fig. 13. Torsional transient dynamic response due to the startup with the linearized flexible coupling characteristic. 


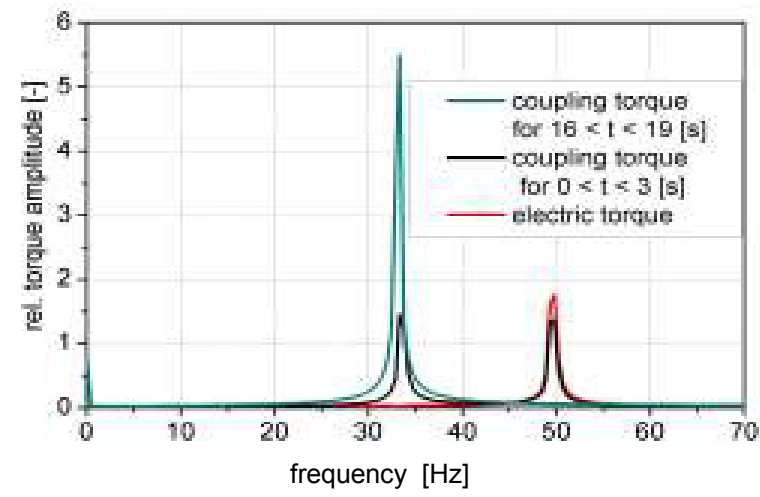

Fig. 14. Amplitude spectrum of the dynamic response during start-up for the system with linearized coupling.

time-history of the dynamic torque transmitted by the flexible coupling has been marked by the green line. From the plots depicted in Fig. 13 it follows that the considered machine was runned-up during ca. $43 \mathrm{~s}$. At the beginning of this process the time-history of the driving motor torque is characterized by the typical, severe fluctuating component of the supply frequency 50 $\mathrm{Hz}$ quickly decaying with time and causing a relatively weak amplification of the dynamic torque transmitted by the flexible coupling and other shaft segments. From the results of the FFT analysis performed for the first three seconds of the run-up and depicted in Fig. 14 it follows that the system dynamic response consists of two fundamental components. The first one of the supply frequency $50 \mathrm{~Hz}$ results from the direct external excitation produced by the driving motor. However, the second one of the first system natural frequency $34.3 \mathrm{~Hz}$ is a typical transient state component induced by the driving torque generated by the asynchronous motor. Then, this component slowly decays with time, but after the $15^{\text {th }}$ second of the start-up it becomes significantly amplified, when the rotating system passes through the rotational speed value ca. $16 \mathrm{rev} / \mathrm{s}$ corresponding approximately to one half of the $1^{\text {st }}$ natural frequency. Since in the considered electromechanical system the rotational speed is affected by the fluctuating component of this frequency, the asynchronous motor electromagnetic torque becomes also additionally affected by this rotational speed fluctuating component. Then, in the case of ca. $34 \mathrm{~Hz}$ the severe resonance is observed, which leads to amplitudes of the flexible coupling dynamic torque exceeding 8 times the nominal torque value, see Fig. 13 and 14. Although these resonant vibrations also quite quickly decay with time, but they are severe enough to damage gradually the drive system elements particularly sensitive to dynamic over-loadings during successively repeated machine start-ups.

In the second computational example the stiffness characteristic of the flexible coupling has been assumed nonlinear according to the plot presented in Fig. 10. Then, the analogous run-up simulation has been carried out and its computational results in time domain are depicted in Fig. 15 in the identical way as in the previously considered case. As it follows from the obtained plots, here the system dynamic response has a completely different qualitative and quantitative

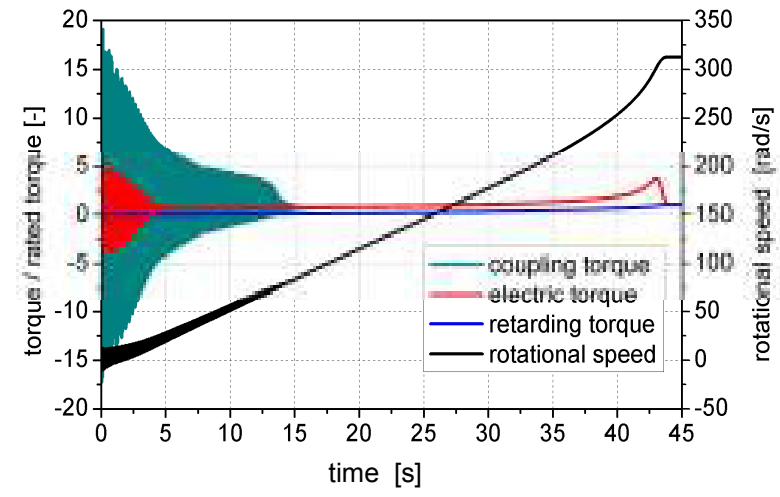

Fig. 15. Torsional transient dynamic response due to the startup with the nonlinear flexible coupling characteristic.

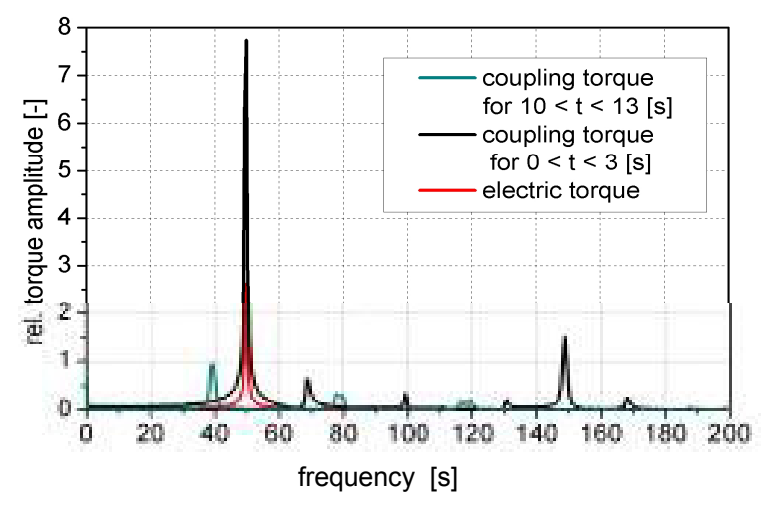

Fig. 16. Amplitude spectrum of the dynamic response during start-up for the system with nonlinear coupling..

character in comparison with that illustrated in Fig. 13. Here, during start-up, together with the rise of the transmitted mean driving torque, the flexible coupling stiffness gradually increases and then the considered drive system changes its first natural frequency from the mentioned above ca. $15 \mathrm{~Hz}$ till more than $50 \mathrm{~Hz}$ at the maximal admissible transmitted torque value. Thus, the drive system consequently changes its dynamic properties, which prevents a possibility of resonance excitation. But at the beginning of the start-up process the dynamic amplification caused by the motor electromagnetic torque is much stronger than in the previous example. Here, the amplitudes of the dynamic torque transmitted by the flexible coupling are more than 15 times greater than the nominal torque value. Hence, despite of a lack of resonance effect, the run-up of the rotor machine equipped by the flexible coupling with such non-linear, progressive characteristics can result in a relatively fast damage of this drive system element expected to transmit dynamic torques exceeding the nominal value only 6 times. From the results of FFT analysis demonstrated in Fig. 16 it follows that during the first 3 seconds of the run-up the excitation by the asynchronous motor with frequency $50 \mathrm{~Hz}$ causes a severe dynamic amplification of the dynamic torque transmitted by the flexible coupling with the fundamental component of the same frequency $50 \mathrm{~Hz}$ as well as with smaller super-harmonics of frequencies ca. $70,100,130,150$ and $170 \mathrm{~Hz}$. However, during the final phase of transient vibrations, i.e. within the range of 10- 
$13 \mathrm{~s}$ of the start-up, the time-history of the coupling dynamic torque consists of the much smaller fundamental component of frequency $\sim 40 \mathrm{~Hz}$ together with the remarkable super-harmonics of frequencies ca. 80 and $120 \mathrm{~Hz}$. According to the above, in order to avoid these detrimental transient oscillations the asynchronous motor of the considered rotor machine must be equipped with a proper inverter enabling us secure and fluent startups by means of driving electromagnetic torques possibly free of forcing fluctuation components.

An operation of majority of asynchronous motor inverters usually reduces to a gradual increase of the supply voltage in order to achieve the required rotational speed at the given loading of the runned-up machine. In the idealized case, when the voltage slowly rises from zero to the nominal value, for the smaller starting torque Variant I the start-up of the considered object lasts almost two times longer than in the previous examples, i.e. without an inverter. But then, the entire run-up process is completely free of torsional oscillations, which follows from the respective plots in Fig. 17 demonstrating the system dynamic response in an identical way as in Figs. 13 and 15. However, in order to overcome some natural static retarding torques in the drive system, when the supply voltage generated by the inverter starts from e.g. ca. $10 \%$ of the nominal value, some slight initial torsional vibrations are excited by the motor. As shown in Fig. 18, they gradually decay with time and when the system with the linear flexible coupling slowly passes the resonance frequency $34.3 \mathrm{~Hz}$,

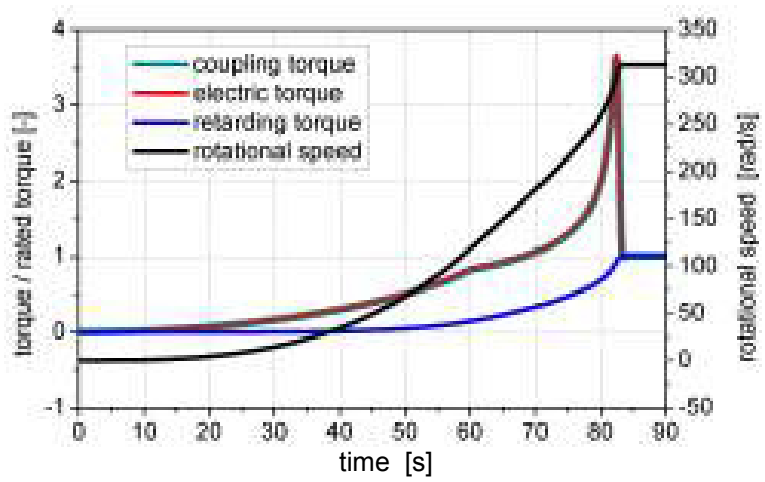

Fig. 17. Torsional transient dynamic response due to the startup with the linearized flexible coupling characteristic for Variant I and zero initial supply voltage.

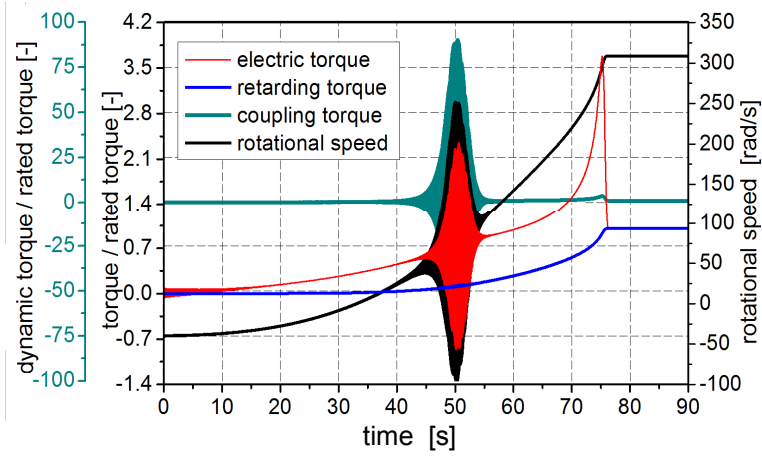

Fig. 18. Torsional transient dynamic response due to the startup with the linearized flexible coupling characteristic for Variant I and non-zero initial supply voltage.

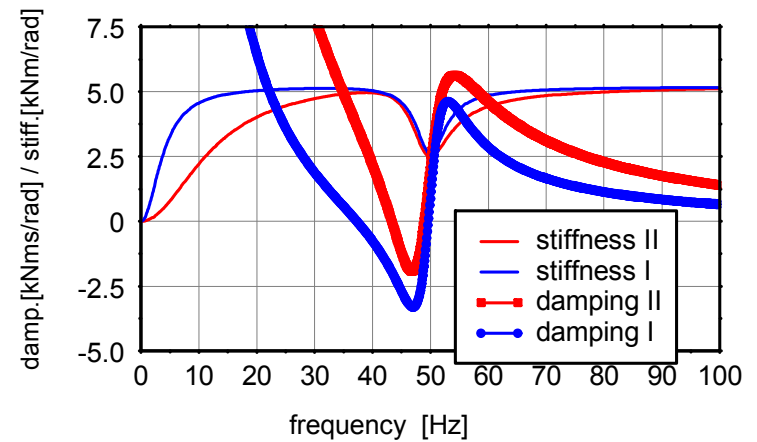

Fig. 19. Characteristics of the rotor-to-stator electromagnetic damping and stiffness for two variants of the asynchronous motor resistances.

huge transient oscillations rapidly occur with amplitudes almost 100 times exceeding the rated torque value. Here, despite of the start-up performed so mildly, such severe overloading can lead to an immediate damage of the flexible coupling. This fact can be explained by not only much slower passage through the resonance zone than in the case illustrated in Fig. 13, but by the very low, even negative electromagnetic damping generated by the asynchronous motor interacting with the torsionally vibrating mechanical system. For the considered here smaller starting motor torque Variant I the negative damping zone is significantly wider than for the greater staring torque version called Variant II. It follows from the respective characteristics of the electromagnetic

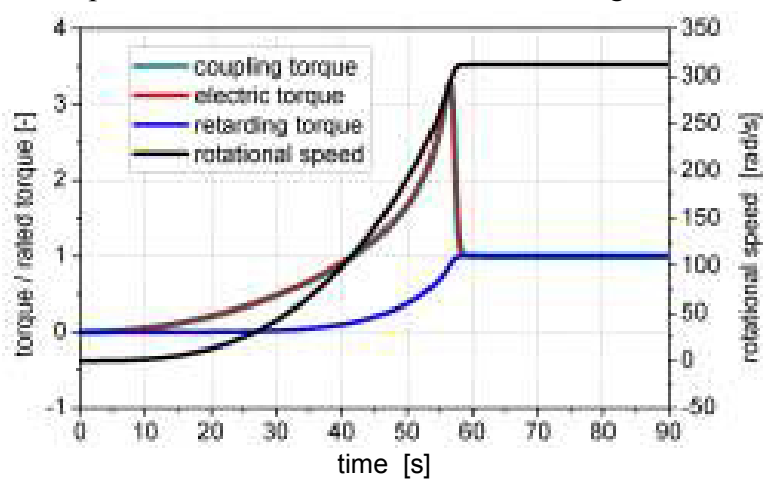

Fig. 20. Torsional transient dynamic response due to the startup with the linearized flexible coupling characteristic for Variant II and zero initial supply voltage.

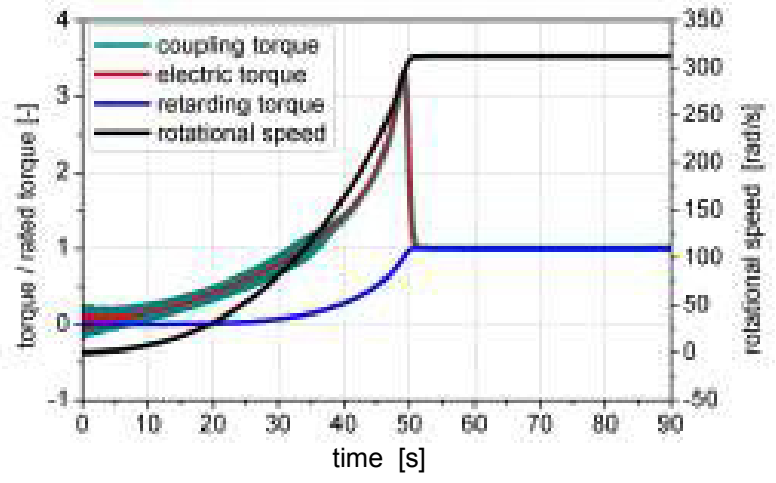

Fig. 21. Torsional transient dynamic response due to the startup with the linearized flexible coupling characteristic for Variant II and non-zero initial supply voltage. 
damping and stiffness depicted in Fig. 19 and determined for this motor using the analytical method described in [8]. Here, for Variant I this zone is contained within the range between ca. 37 and $49 \mathrm{~Hz}$. Hence, the electromagnetic damping coefficient values in the vicinity of the resonant frequency $34.3 \mathrm{~Hz}$ are very small. Then, in the presence of so common, relatively low damping magnitude in torsionally vibrating drive systems such dangerous resonances can be easily excited. However, for Variant II corresponding to the greater starting motor torque, see Fig. 12, the negative damping zone is more narrow and placed between ca. 44 and $48 \mathrm{~Hz}$. Furthermore, as shown in Fig. 19, since the electromagnetic damping coefficient values in the vicinity of $34.3 \mathrm{~Hz}$ are significantly greater, possibilities of resonance excitation are appropriately smaller. According to the above, when the inverter is used, the asynchronous motor with greater starting torque seems to be more convenient for start-ups of the considered rotating machine. This fact has been confirmed by the run-ups with zero- and non-zero initial voltage values. The dynamic responses induced by these start-ups are presented respectively in Figs. 20 and 21. Here, in the case of zero-initial supply voltage the completely oscillation-free response is observed, similarly as that obtained for motor Variant I and depicted in Fig. 17. However, during start-up with the initial voltage equal to $10 \%$ of the nominal value from the beginning of the process some mild transient vibrations occur, which gradually decay with time, as shown in Fig. 21.

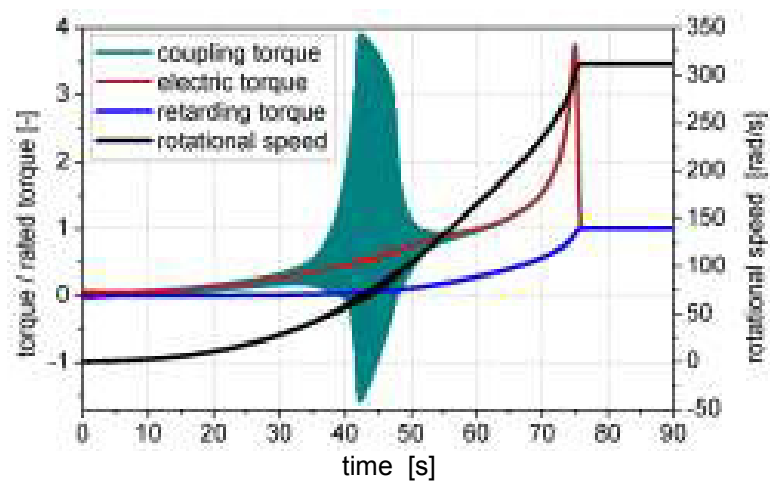

Fig. 22. Torsional transient dynamic response due to the startup with the non-linear flexible coupling characteristic for Variant I and non-zero initial supply voltage.

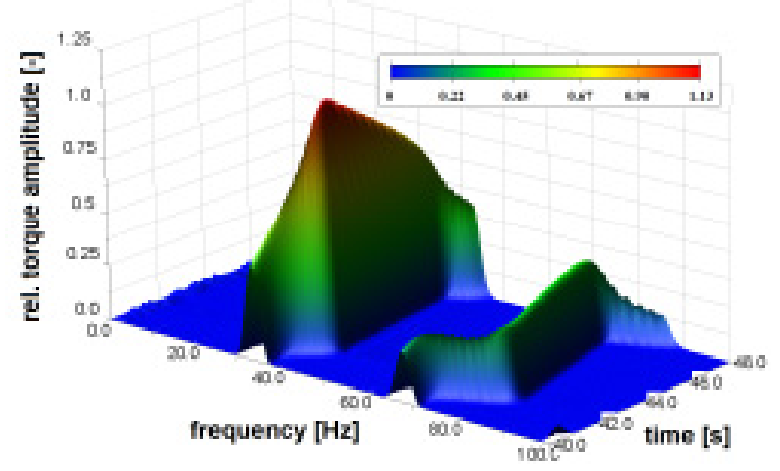

Fig. 23. Waterfall diagram for the dynamic torque amplitudes in the non-linear flexible coupling due to the start-up for Variant I and non-zero initial supply voltage.
When in the considered rotor machine drive system the flexible coupling with non-linear characteristic is assumed, for the asynchronous motor Variant I with the smaller starting torque and for the zero initial supply voltage the identical vibration-free dynamic response has been obtained as in the case of the linear coupling depicted in Fig. 17. However, during the run-up with the initial voltage equal to $10 \%$ of the nominal value remarkable transient vibrations are induced between the $30^{\text {th }}$ and $55^{\text {th }}$ second of the process, as illustrated in Fig. 22. These oscillations are not so severe as those resonant ones observed during start-up of the system with linear flexible coupling. Here, due to the gradual stiffening of the flexible coupling with non-linear characteristic the transient oscillations increase their frequency in a piecewisely linear way within ca. $30-50 \mathrm{~Hz}$, as demonstrated by means of the waterfall diagram in Fig. 23. Moreover, in this diagram the first super-harmonic component of the non-linear vibrations is observed.

Variant II of the asynchronous motor with the greater starting torque enables us much faster run-up. For zero initial supply voltage it also results in oscillation-free dynamic response, identical with that presented in Fig. 20 for the drive system with the linear flexible coupling. For the non-zero initial supply voltage equal to $10 \%$ of the nominal value the quicker rotational speed increase associated with the simultaneous synchronous rise of excitation frequency according to Eq. (3) makes an induction of severe transient oscillations difficult. Thus, during the start-up in such conditions the obtained

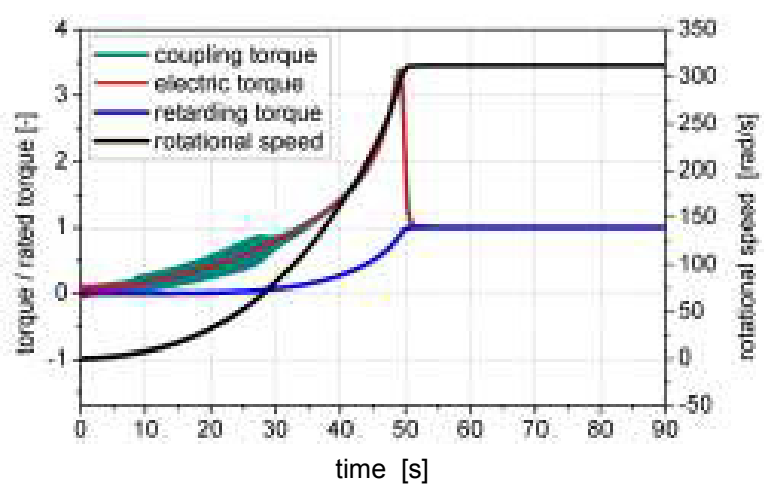

Fig. 24. Torsional transient dynamic response due to the startup with the non-linear flexible coupling characteristic for Variant II and non-zero initial supply voltage.

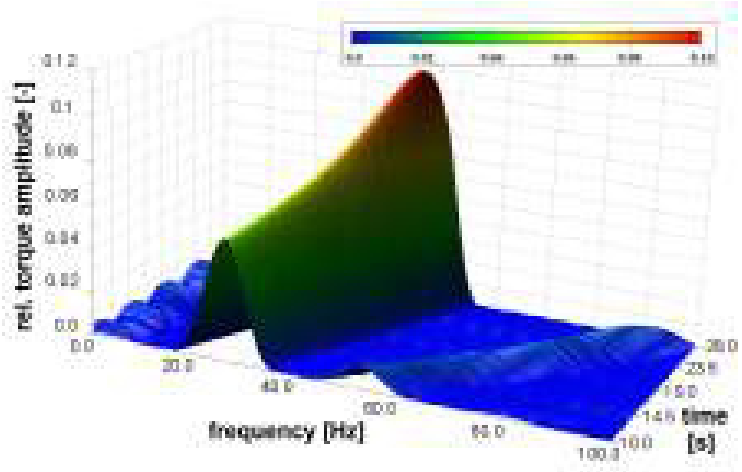

Fig. 25. Waterfall diagram for the dynamic torque amplitudes in the non-linear flexible coupling due to the start-up for Variant II and non-zero initial supply voltage. 
dynamic response is characterized by very mild transient torsional vibrations transmitted by the flexible coupling, which are observed between the $10^{\text {th }}$ and $28^{\text {th }}$ second of the process, as shown in Fig. 24. But here, the oscillation amplitudes are significantly smaller than these observed in the case of slower run-up using Variant I of the motor starting torque. From the respective waterfall diagram depicted in Fig. 25 it follows that due to the gradual stiffening of the non-linear coupling the fundamental frequency of transient oscillations increases linearly within the abovementioned ca. $10-28 \mathrm{~Hz}$ as well as its first super-harmonic is also remarkable.

\section{Final remarks}

In the paper dynamical aspects for design and maintenance of the selected group of machines applied in the mining industry are considered. These machines and devices are driven by the asynchronous motors and they are characterized by rotational motions of their fundamental working tools. Thus, the dynamic investigations have been performed in the way typical as for the rotating machinery, i.e. in the form of bending (lateral) and torsional vibration analyses. Moreover, in these studies there was also investigated a mutual interaction between the mechanical and electrical part of the considered objects. Contrary to the traditional engineering approaches usually applied so far for design and maintenance of these machines, for the computations carried out here the advanced mechanical and electromechanical models and computer techniques have been used. By means of these modern computational tools the main attention was focused on qualitative aspects of dynamic behaviours typical for beater mills and crushers as well as for blowers and compressors.

From the results of bending vibrations analyses performed for the listed above machines characterized by heavy beater wheels or impellers designed in the form of overhung rotors it follows that their dynamic loadings caused by unbalances turned out to be particularly dangerous for fatigue life of their the most responsible elements. These unbalances are more dangerous than dynamic loadings associated with natural working processes, since they are caused not only by unavoidable manufacturing inaccuracies, but first of all by gradual and progressive material, thermal or erosive damages of working tool surfaces. The bending vibrations of the rotor-shafts of these machines induced by these unbalances can be a source of too fast material fatigue often leading to cracks and even to catastrophes, of dynamic over-loadings of bearing supports usually being transmitted to the environment as well as of harmful noise generation. According to the above, these detrimental and dangerous effects have to be thoroughly taken into consideration during routine design phases of these machines as well as they should be on-line monitored during regular operation in order to asses a degradation level of the working tools.

The torsional vibrations have been investigated here as entirely associated with electrical vibrations in driving motor windings in the form of electromechanical interaction analysis. From the results of performed computations it followed that steady-state operation conditions of the considered machines are not a source of essential torsional vibrations, contrary to quite frequently repeated start-ups of these devices, which can cause very severe or even dangerous transient oscillations. Here, mutual interdependencies between the electrical parameters of the driving motor and dynamic properties of the driven mechanical part significantly influence magnitudes of these vibrations. It turned out that the most important role play stiffness characteristics of the flexible couplings, an ability of negative electromagnetic damping generation by the asynchronous motors as well as a character of motor supply voltage control during start-up processes. First of all, in order to avoid severe transient torsional vibration amplitudes the asynchronous motors with inverters should be applied to control properly the supply voltage influencing runs of the driving motor torques. Then, start-ups usually last longer, but without remarkable oscillations, if system parameters had been properly selected before. Namely, the flexible couplings with non-linear characteristics seem to be more convenient, since they have an ability to mistune possible resonances. Next, the asynchronous motors with greater starting torques can assure shorter start-ups in time and thus also faster passages through eventual resonance frequency zones. Moreover, such asynchronous motors are able to generate less negative electromagnetic damping which is very dangerous from the viewpoint of an excitation of transient resonances and operational instabilities.

\section{References}

1. R. Gasch, R. Nordmann, H. Pfützner, Rotordynamik (Springer-Verlag, 2002)

2. G. Genta, Dynamics of Rotating Systems (Springer Science+Business Media, 2005)

3. M.I. Friswell, J.E.T. Penny, S.D. Garvey, A.W. Lees, Dynamics of Rotating Machines (Cambridge Aerospace Series) (Cambridge University Press, 2010)

4. ISO 1940/1, Balance Quality Requirements of Rigid Rotors (Int. Org. for Standardization, 2003)

5. ISO 11342, Mechanical Vibration - Methods and Criteria for the Mechanical Balancing of Flexible Rotors (Int. Org. for Standardization, 1998)

6. K. L. Shi, T. F. Chan, Y. K. Wong, L. S. Ho, Int. J. of Electrical Engineering Education, 36 (1999)

7. D.C White, H.H. Woodson, Electromechanical Energy Conversion (Wiley, 1959)

8. T. Szolc, R. Konowrocki, M. Michajłow, A. Pręgowska, Mech. Syst. and Signal Proc., 49 (2014) 